\title{
Factores que intervienen en riesgo suicida y parasuicida en jóvenes Chilenos
}

\section{Factors involved in suicidal and parasuicidal risk in Chilean youth}

\section{Alexis Soto Salcedo}

Universidad Mayor, Chile

ORCID: https://orcid.org/0000-0002-1304-4438

Patricia Villaroel Grüner

Universidad Mayor, Chile

ORCID: https://orcid.org/0000-0003-2650-9772

Alex Véliz Burgos

Universidad de Los Lagos, Chile

ORCID: https://orcid.org/0000-0003-1371-9041

Received 03-24-20 Revised 05-30-20 Accepted 08-08-20 On line 10-27-20

*Correspondence

Email: alexis.soto@umayor.cl
Cite as:

Soto, A., Villarroel, P., \& Véliz, A. (2020). Factores que intervienen en riesgo suicida y parasuicida en jóvenes Chilenos. Propósitos y Representaciones, $\quad 8(3)$ e672.

http://dx.doi.org/10.20511/pyr2020.v8n3.672 


\section{Resumen}

El establecimiento de factores intervinientes en riesgo suicida y parasuicida trae consigo una serie de conceptualizaciones que este articulo contempla en primer lugar y que posteriormente presenta de forma ordenada y simple, gracias a una revisión sistemática que da cuenta de estudios realizados principalmente en el continente americano, y especialmente considerando la dinámica chilena en este aspecto. Como principales resultados se establece la explicitación de factores personales, familiares y de contexto tanto sociales como comunitarios, que son revisados desde diversos autores y que sin duda aportan por un lado a la comprensión primaria de riesgo suicidio y de paso se convierten en un insumo fundamental para el diseño y construcción de programas de prevención de la conducta suicida.

Palabras claves: Riesgo suicida, suicidio, conducta parasuicida

\section{Summary}

The establishment of intervening factors in suicide and parasuicide risk brings with it a series of conceptualizations that this article considers and presents in an orderly and simple way, thanks to a systematic review that accounts for studies carried out mainly in the American continent, and especially considering the Chilean dynamics in this regard. As main results, it establishes the explicitness of personal, family and context factors, both social and community, that are reviewed by various authors and that undoubtedly contribute on the one hand to the primary understanding of suicide risk and, incidentally, become a fundamental input for the design and construction of programs to prevent suicidal behavior.

Keywords: Suicidal risk, suicide, parasuicidal behavior

\section{Introducción}

El fenómeno del suicidio es altamente complejo pues si bien existen algunos indicadores que permiten realizar una comprensión básica del tema, existe también un marco inaccesible de información que rodea a la persona que ha consumado el suicidio y al cuál es imposible acceder. Diversos investigadores del mundo público y privado han destinado esfuerzos para conocer de mejor forma la manera de incidir en los procesos primaros de ideación suicida con el sentido de evitar las conductas autolíticas exitosas y de esa forma realizar una contribución al bienestar de las familias que se ven enfrentadas a lidiar con este tema.

Al revisar la literatura, es posible evidenciar que existen diversos factores que inciden en la presentación del suicidio, se han sistematizado algunos factores de riesgo que parecieran ser comunes y que al identificarlos permiten tener nociones respecto de ámbitos que son importante de cuidar y en los cuáles es posible generar acciones de intervención que apunten a garantizar una suerte de equilibrio psíquico que favorezca una opción para la vida. De todas formas, el postmodernismo, las conflictivas sociales y la situación actual de muchos de los países que manifiestan estados de descontento y aflicción llevan a que cada vez sea más complejo el abordaje del suicidio. Las sociedades modernas, con sus ritmos frenéticos, nos contagian el ansia por la satisfacción inmediata, enaltecen el bienestar y potencian el consumismo compulsivo, incluyendo el de la salud como producto (Navarrete, Herrera y León, 2019).

Hoy en día es relevante estar en continua revisión de estos estudios a objeto de levantar información nueva, o precisar respecto de ámbitos o áreas de la vida de las personas que 
parecieran ser más sensibles o verse vulneradas con mayor facilidad y que llevan a instalar el suicidio como una alternativa. Por esta razón se realiza una revisión sistemática cuyo objetivo es establecer factores sociodemográficos y factores de protección y de riesgo suicida y parasuicida de jóvenes Chilenos, realizando una búsqueda exhaustiva de la literatura existente.

\section{Desarrollo del tema}

La conducta suicida es una causa importante de morbi mortalidad a nivel mundial. Por esta razón, la medición del riesgo suicida y la identificación de las personas en riesgo de un intento suicida son tareas prioritarias. El suicidio constituye un problema mundial de salud pública, realizándose esfuerzos de prevención, especialmente en población adolescente (Silva, Valdivia, Vicente, Arévalo, Dapelo, \& Soto, 2017).

A lo largo de la vida, diversos factores como el estrés y la forma de enfrentarlo, el dilema que plantea la esperanza y la desesperanza y la falta de autoestima, los procesos de autodestrucción por el consumo de drogas, la delincuencia, la sexualidad desprotegida, entre otros, se relacionan con el suicidio (González-Forteza, García, Medina-Mora, \& Sánchez, 2013). Partiendo de un adecuado conocimiento de los factores asociados con las conductas suicidas, se pueden desarrollar estrategias que permitan su prevención y la reducción de la mortalidad y las secuelas generadas por esta causa. Se deberían encontrar aspectos que el clínico pueda explorar y manejar de manera adecuada, con miras a reducir la mortalidad y las secuelas por dicha causa (Sánchez, Cáceres, \& Gómez, 2002).La ideación suicida, el intento suicida y el suicidio no son fenómenos inusuales en el contexto universitario (Restrepo, et al, 2018) y los factores de riesgo asociados a la conducta suicida en la infancia y adolescencia han sido numerosos, encontrándose en ocasiones datos contradictorios.

Algunos de esos datos tienen que ver con factores como violencia y maltrato vivenciado por los adolescentes. El hallazgo de antecedentes de maltrato infantil está a favor de las hipótesis que involucran este antecedente en la etiología de los trastornos psiquiátricos, especialmente en los correspondientes al espectro depresivo. Otro aspecto que resulta relacionado con la ideación suicida fue la exposición a información sobre el suicidio de otros (Sánchez et al., 2002). Además de lo anterior la presencia de violencia verbal se asocia a conducta suicida de forma independiente a la presencia de violencia física y/o sexual, lo cual sugiere que la percepción de agresión verbal en el ámbito doméstico puede llegar a tener tanto o mayor impacto sobre el desarrollo de tendencias autodestructivas en los menores que la violencia física o sexual, aunque el número tan pequeño de estos últimos casos hace difícil establecer su verdadero peso como factores de riesgo independientes.

Cuando se analizan los eventos vitales estresantes, se encuentra un mayor nivel de ideación suicida en estudiantes que manifiestan haber vivenciado eventos tales como cambiar de grupo de compañeros, vivir alejado de la familia, tener peleas y discusiones graves con un ser querido, tener un familiar con enfermedad grave mortal, seres queridos con problemas emocionales o psicológicos, vivenciar maltrato físico intrafamiliar, problemas con drogas o alcohol y verse afectado por un desastre natural (Siabato, y Salamanca, 2015). 


\section{Comprensión del suicidio}

El comportamiento suicida se refiere a las cogniciones y actividades de las personas que buscan quitarse la vida, incluyendo pensamientos, acciones u omisiones. (Jans, Vloet, Taneli, \& Warnke, 2017). Se puede entender el comportamiento suicida como un proceso complejo que puede incluir ideaciones suicidas, amenazas, gestos e intentos, hasta desembocar en el suicidio propiamente dicho, aunque puede ocurrir suicidio sin ideación previa, así como ideaciones que no lleven a la muerte autoinfligida. (Vélez, Maldonado y Rivera, 2017).

El conocimiento acerca del comportamiento suicida ha aumentado enormemente en los últimos decenios. La investigación ha mostrado la importancia de la interacción entre factores biológicos, psicológicos, sociales, ambientales, culturales y las condiciones económicas desfavorables que intervienen en la determinación de los comportamientos suicidas (Corona, Hernández y García, 2016; Cañon et al., 2018).

Barrios et al. (2017) señala que el comportamiento suicida como fenómeno, se puede comprender en dos polos de entendimiento, por un lado, se comprende el mismo como un problema individual, producto de una enfermedad mental que se facilita por otros factores de riesgo interpersonales, familiares y sociales, y se interviene principalmente por la psiquiatría y la psicologia; y por el otro, como un comportamiento individual pero que se comprende en relación con las condiciones históricas, culturales, económicas, políticas y sociales dentro de contextos y periodos específicos.

Miranda (2009) planteó cinco etapas que pueden presentarse en el comportamiento suicida, sin que necesariamente tengan que ser secuenciales. Estas etapas son (1) Ideación suicida pasiva, (2) contemplación activa del propio suicidio, (3) planeación y preparación, (4) ejecución del intento suicida, (5) el suicidio consumado.

Considerando estas etapas se podría comprender entonces la ideación suicida, etapa de vital importancia como factor predictor para llegar al suicidio consumado, como aquellos pensamientos intrusivos y repetitivos sobre la muerte autoinfligida, sobre las formas deseadas de morir y sobre los objetos, circunstancias y condiciones en que se propone morir (Nieto et al., 2018).

El intento suicida en cambio se refiere a las fórmulas para operacionalizar el pensamiento y en ocasiones tiene a confundirse con la autolesión no suicida. Para clarificar esta distinción, Jans et al. (2017) presenta un cuadro que explica las diferencias entre una y otra

Tabla 1

Diferencias entre un intento suicida y una autolesión no suicida (Jans et al., 2017)

\section{Intento suicida}

- Hay la intención de acabar con la vida

- Puede ser impulsivo, pero en la mayoría de los casos existe un sentimiento crónico de desesperanza o soledad

- Son frecuentes las formas más graves de las conductas de autoagresión (p.ej.,

\section{Autolesión no suicida (Parasuicidio)}

- No hay intención suicida

- El estado emocional es de ira aguda, desesperación o malestar intolerable

- Son frecuentes las formas de conducta autodestructiva menos graves $y$ mayoritariamente no amenazantes para la vida 
autoenvenenamiento, ahorcamiento, saltar de gran altura, uso de armas de fuego) (p.ej., lesiones cutáneas por mordeduras, cortes, quemaduras o congelamiento)

- Normalmente, la persona es consciente de que la conducta puede causar lesiones graves, pero no amenaza la vida.

- Existe un claro riesgo de que los intentos suicidas se repitan, pero menos frecuentemente que las conductas autolesivas no suicidas.

- Las autolesiones recurrentes son frecuentes

\section{Intento suicida Autolesión no suicida ("parasuicida")}

El suicidio consumado se refiere al hecho de conseguir el objetivo de terminar con la propia vida a través de algún método o formula planeada. Navarro-Gómez (2017), realiza una revisión de la literatura disponible respecto del suicidio en jóvenes de España y logra dar cuenta del modus operandi para consumar el suicidio, en dicho estudio reporta que el sistema elegido por la gran mayoría de jóvenes de ambos sexos entre los 15 y los 29 años es el ahorcamiento, la estrangulación o sofocación (45.85\%), seguido de otras formas como lanzarse al vacío (30.56\%). A partir de aquí, encontramos diferencias entre los sexos: mientras que para los chicos el tercer método de suicidio preferido es auto dispararse con armas de fuego diferentes de rifle, escopeta y arma larga o arma corta y otras armas no especificadas, seguido del consumo de drogas y otras formas de suicidio no especificadas o inhalación de gases, para el sexo femenino el tercer método utilizado es el envenenamiento con drogas, medicamentos o sustancias biológicas, seguido del ahogamiento y otras causas no especificadas.

\section{Método}

Se realiza una revisión exhaustiva entre los meses de octubre del año 2019 y Febrero del año 2020 usando como motores de búsqueda Science Direct, scopus, Education Source, Metabuscador Ufro, Google Scholar, Web of Science, en las bases de datos de Scopus, WOS, Science Direct, Dialnet, EBSCOhost. Se revisan artículos tanto en inglés como en español considerando principalmente aticulos que estuviaran focalizados en población adolescente. Para ello se busca información sin filtrar un país o continente específico, pero se intenta identificar experiencias latinoamericanas y principalmente chilenas respecto del tema.

Los criterios usados para incluir artículos fueron (a) que estuviera enfocado en población adolescente, y (b) que estableciera como palabra clave de búsqueda: suicidio, comportamiento suicida, riesgo suicida, riesgo suicida en adolescentes. Se realizó una primera selección revisando resúmenes y abstract y se construyó una planilla Excel que recoge elementos esenciales de cada revisión descargando los artículos seleccionados para la construcción de este artículo que servirá además como cribado para la profundización en materias más específicas de esta temática.

Finalmente se seleccionaron 87 artículos para revisión. 
Resultados

\section{Factores Sociodemográficos}

El suicidio presenta un desafío importante para la salud pública en los Estados Unidos y en el mundo. Contribuye a la muerte prematura, la morbilidad, la pérdida de productividad y a costos en términos de atención médica. En el 2015, el suicidio fue responsable de 44193 muertes en los Estados Unidos, lo cual se traduce a aproximadamente 1 muerte cada 12 minutos (Stone et al., 2017).

El comportamiento suicida en la población joven universitaria también es un problema relevante en América Latina. En Puerto Rico el suicidio es uno de los problemas más alarmantes de salud. Según el Departamento de Salud de Puerto Rico se han registrado más de 4,500 muertes por suicidio en los últimos 15 años. (Vélez, Maldonado y Rivera, 2017). En este mismo sentido los autores desarrollaron un estudio descriptivo aplicando un cuestionario a 507 jóvenes universitarios del área sur de Puerto Rico El 9.9 \% indicó haber realizado durante su vida algún acto con la intención de quitarse la vida, mientras que el $8.5 \%$ afirmó que tuvo alguna ideación suicida en el último año.

A su vez, Borges Orozco, Benjet, \& Medina-Mora (2010) realizan una revisión de la mortalidad por suicidio de 1970 hasta 2007 en México, por medio de encuestas transversales, desde allí obtiene que el suicido ha crecido $275 \%$. Actualmente el suicidio se incrementa en el grupo de 15-29 años de edad. La prevalencia de por vida de ideación suicida en adultos de 18 a 29 años fue de $9.7 \%$ y $3.8 \%$ reportaron intento de suicidio. Entre los habitantes de la República mexicana, 6.601.210 tuvieron ideación suicida en los últimos 12 meses, 593.600 personas intentaron suicidarse y 99.731 utilizaron servicios médicos como consecuencia de un intento de suicidio

En Cuba el suicidio es la novena causa de muerte en el cuadro de mortalidad general; ocupa la tercera causa de muerte en el grupo de 10-19 años y el 4to lugar en los grupos de 5-14 años y de 15-49 años, incrementándose las tasas a partir de los mayores de 60 años de 24,1 por 100000 habitantes hasta 48,3 por 100000 habitantes en los mayores de 80 años en el trienio del 2008-2010. (Corona, Hernández y García, 2016).

En Colombia mientras tanto un estudio desarrollado por Cañon et al. (2018) encontró que en una aplicación preliminar la escala de Riesgo Suicida de Plutchik, la encuesta mito o realidad, escala de Asertividad de Rathus, y escala de autoestima de Rosenberg, apareció un 42,7\% de riesgo suicida, en mayor proporción 56,9\% de autoestima baja, en mayor proporción $68,9 \%$ de asertividad confrontativa, y 2,9 en el cuestionario sobre mitos alrededor del suicido.

Romo y Kelvin (2016) evaluaron a los predictores de suicidio entre adolescentes en latinoamerica usando el Cuestionario Global de Salud Escolar (GSHS) por sus siglas en inglés y encontraron que Bolivia tiene el mayor porcentaje $(20,9 \%)$ de estudiantes reportando al menos 1 intento de suicidio.

En Perú, se realizó un estudio descriptivo-correlacional con 354 estudiantes del nivel secundaria de la institución educativa Mariscal Castilla cuyos resultados indican que dentro de los factores más relevantes para la ocurrencia del suicidio se encuentran los problemas familiares, el acoso escolar, la situación económica, conductas y antecedentes familiares de suicidio, las 
dificultades de aprendizaje y bajo rendimiento escolar y algunos otros con menor incidencia estadística (Marín y Jorka, 2018).

\section{Suicidio en Chile}

En la región de las américas, según la Organización Panamericana de la Salud (2014), los países con las tasas más altas de suicidio son: Guyana (26.2 por 100 mil habitantes), Surinam (23.3), Uruguay (14.2), Chile (11.2), Trinidad y Tobago (10.7), Estados Unidos (10.1), Cuba (9.9) y Canadá (9.7).

Según la Organización para la Cooperación y el Desarrollo Económicos (OCDE), Chile es el país donde más ha aumentado el suicidio, después de Corea del Sur, con una tasa de 13,3 muertes por cada 100.000 habitantes, con un crecimiento de $90 \%$ entre los años 1990-2011 (Ardiles- Irarrázaval, Alfaro-Robles, Díaz-Mancilla, Martínez-Guzmán, 2018).

Chile es un país con altas tasas de enfermedades mentales, cuyos costos directos e indirectos afectan negativamente a los individuos que las padecen, sus familias y la sociedad en su conjunto, aumentando -por ejemplo- los costos y usos de los servicios generales de salud. Si bien el Ministerio de Salud ha reconocido crecientemente la relevancia de los problemas de salud mental, el financiamiento de la salud mental no ha aumentado según los objetivos propuestos. Los planes de salud mental implementados en la última década no han reducido las altas tasas de enfermedades mentales. De acuerdo a datos del Ministerio de Educación de Chile (2019) desde el año 2006 al año 2016 se presenta una tasa promedio de 10,6 y 15,9 en rangos de edad de 15 a 19 años y de 20 a 24 años respectivamente. Ambos rangos se corresponden a las edades más comunes que presentan los estudiantes universitarios que ingresan habitualmente a los 17 o 18 años y egresan cerca de los 24 años.

En este sentido, un estudio realizado en la Universidad Austral de Chile demostró que el $13,7 \%$ de los estudiantes cumple con criterios para una depresión mayor y si se considera cualquier tipo de depresión un 26,9\% cumple con los criterios (según escala PHQ-9). En tanto que un $10,4 \%$ cursa con un trastorno bipolar (según escala MDQ). Es importante destacar que el $5,3 \%$ de los estudiantes presenta puntuaciones elevadas de desesperanza, lo que sugiere un riesgo moderado a severo de cometer suicidio y/o autolesiones no fatales (según escala de desesperanza de Beck). (Baader et al, 2014) El mismo estudio presenta con relación al riesgo de suicidio, que las personas con criterios para depresión mayor se asocian a puntuaciones elevadas de desesperanza. La desesperanza es un factor de riesgo para suicidio y autolesiones no fatales, por lo que las puntuaciones obtenidas son un predictor útil de posible suicidio. Esto viene a reafirmar la complejidad del cuadro depresivo y su asociación con el riesgo suicida. (Baader et al., 2014).

Otro estudio examina variables sociodemográficas, familiares, abuso de sustancias, suicidalidad, autoestima, depresión, desesperanza, funcionalidad familiar, impulsividad y acontecimientos vitales. A partir de 919 participantes se obtuvo una prevalencia de vida del intento de suicidio de 14.3\%. Los resultados de los auto-informes que se aplicaron indican que la mayoría de los factores de riesgo analizados fueron asociados al intento de suicidio. Sin embargo, en el análisis multivariado solamente ingresaron el consumo de tabaco, la ideación suicida tanto reciente como antigua y los acontecimientos vitales (Silva et al., 2017). 


\section{Factores de Riesgo y Protección de la Conducta Suicida}

Los factores de riesgo señalados por U.S. Office of the Surgeon General, National Action Alliance for Suicide Prevention (2012) y la World Health Organization (2014) incluyen, por ejemplo:

- En el nivel individual: antecedentes de depresión y de otras enfermedades mentales, desesperanza, abuso de sustancias, ciertas afecciones, intentos de suicidio anteriores, ser víctima o perpetrador de violencia, y determinantes genéticos y biológicos.

- En el nivel relacional: relaciones altamente conflictivas o violentas, sensación de aislamiento y de falta de apoyo social, antecedentes de suicidio de un familiar o ser querido, estrés financiero o laboral.

- En el nivel comunitario: inadecuada conexión con la comunidad, barreras para la atención médica (por ejemplo, falta de acceso a proveedores y medicamentos).

- En el nivel social: disponibilidad de medios letales para cometer el suicidio, representaciones del suicidio en los medios de manera no segura, estigma relacionado con buscar ayuda y con la enfermedad mental

Para profundizar en los factores personales de riesgo para una conducta suicida es posible mencionar que existen ciertos factores que han sido mencionados en numerosos estudios como posibles desencadenantes de conductas suicidas en los adolescentes. Dentro de estos se incluyen aspectos tales como la edad, el género y el estado civil, y otros relacionados con los rasgos de personalidad, la orientación sexual y las enfermedades físicas y mentales preexistentes (Ruiz y Chaves, 2017). Un estudio de Arce y Arturo (2017) señala que los estudiantes que consumieron bebidas alcohólicas, que presentaron consumo de tabaco, que fueron víctimas de bullying y reportaron haber sido atacados físicamente, mostraron tener mayores posibilidades de presentar intento de suicidio.

Montes y Tomás (2016) indican que, si bien no es posible inferir asociaciones causales, los resultados obtenidos por ellos advierten que la autoestima sería el principal factor interviniente en la aparición del riesgo suicida.

Otro factor personal asociado a la conducta suicida es la presencia de algún trastorno psiquiátrico, investigaciones previas realizadas por Borges et al. (2010) señalan que alrededor de 75\% de los casos de intento de suicidio de la Encuesta Nacional de Epidemiología Psiquiátrica Mexicana reportaron antecedentes de algún trastorno psiquiátrico y en el caso de los adolescentes de la región metropolitana del Distrito Federal de México, se encontraron antecedentes de algún trastorno mental en $85 \%$ de aquellos con un intento de suicidio.

Dentro de los factores familiares asociados con la aparición de conductas suicidas en adolescentes, se ha investigado la dinámica del funcionamiento familiar como un aspecto determinante (Ruiz y Chaves, 2017). Un ejemplo de ello es la asociación encontrada entre abuso sexual en la infancia e ideación suicida. Tabares, Villa, y Rendón, (2019) indican que las dinámicas familiares conflictivas y el tipo de autoridad que los padres ejerzan con sus hijos pueden ser factores de riesgo para futuras problemáticas sociales a las que se exponen los jóvenes, por lo que probablemente no tendrán las suficientes herramientas para resolver los conflictos que les presenta el medio social. En varias investigaciones se ha encontrado que el modelo parental autoritario, pero con un equilibrio entre el afecto y el control, permite que el niño tenga mejores habilidades interpersonales, bienestar emocional y menos riesgos de caer en problemas de drogadicción, suicidio, entre otras. 
Complementando lo anterior, los resultados del estudio desarrollado por Nova, Hamid, y Daulima (2019) encontraron cinco temas que vinculan la familia con los intentos de suicidio: los intentos de suicidio son una carga compleja para las familias, los cambios de comportamiento como un signo de suicidio, la preocupación como una forma de apoyo familiar y comunitario, las percepciones familiares sobre las causas y consecuencias del intento de suicidio y las estrategias de supervivencia de las familias superando el impacto del intento de suicidio.

Jans et al. (2017) hace énfasis en que la psicopatología paterna y los antecedentes de conducta suicida en la familia se asocian a un mayor riesgo de suicidio en la descendencia. La propensión al suicidio en familias puede ser independiente de una historia familiar de enfermedades psiquiátricas. Los niños de padres con trastornos del estado de ánimo muestran más intentos de suicidio, cuando hay antecedentes de suicidio de los padres, en comparación con los hijos de padres sin historia de intentos de suicidio.

Si consideramos los factores de riesgo sociales y comunitarios es relevante señalar que el contexto social donde se desenvuelve el joven adolescente puede influir en gran medida en la aparición de conductas suicidas sobre todo aquellos elementos relacionados al ambiente escolar y universitario (Vélez, Maldonado y Rivera, 2017; Ruiz y Chaves, 2017).

En Colombia la discriminación social, el secuestro, la tortura y las heridas de guerra son condiciones que, de manera general, conducen a situaciones de estrés en los adolescentes que favorecen el desarrollo de comportamientos autodestructivos tales como el intento de suicidio y el suicidio consumado (Ruiz y Chaves, 2017).

El factor de imitación o repetición podría también estar vinculado a la influencia social que tiene el suicidio. De hecho, se calcula que por cada suicidio consumado en España se realizan entre 20 y 30 intentos suicidas, con lo que el riesgo de repetición es importante. Hasta un 35-50\% repiten el intento y entre aquellos que han cometido suicidio un $40 \%$ han realizado un intento previo y por ello el intento se convierte en un riesgo elevado de posterior consumación (Hernández-Castillo, 2016).

\section{Factores protectores de la conducta suicida}

U.S. Office of the Surgeon General, National Action Alliance for Suicide Prevention. (2012) y la World Health Organization. (2014) señalan que al igual que los otros comportamientos humanos, el suicidio no tiene una causa determinante única. Ocurre como respuesta a la interacción de varias influencias biológicas, sicológicas, interpersonales, ambientales y sociales, generalmente, a lo largo del tiempo, por lo que existen factores de riesgo y de protección en cada uno de estos niveles.

A nivel personal y tal como la autoestima puede ser un factor de riesgo, de igual forma puede ser un factor protector de la vida. Según Sánchez, Muela y García, (2018) pareciera ser que la autoestima podría ser igualmente una variable moduladora de las tentativas suicidas

Además de las variables personales, la familia también cumple un rol fundamental. Teniendo en cuenta lo antes mencionado, algunos investigadores han discutido sobre cuáles pueden ser las funciones esenciales de la familia, exponiendo, por ejemplo, que las principales características de una familia funcional son las jerarquías claras, los límites claros, los roles definidos, la comunicación abierta y explícita, la afectividad y cooperación entre los miembros y la capacidad de adaptación al cambio (Ruiz y Chaves, 2017). 
Para finalizar, un factor adicional que vale la pena mencionar como determinante para el desarrollo de conductas suicidas es la ausencia de redes de apoyo social. (Ruiz y Chaves, 2017). Así, el apoyo social podría estar implicado y tal vez actuar como factor modulador entre las ideas de suicidio y la ejecución real del intento, sobre todo, porque sentir pertenencia, intimidad, consuelo, escucha o ánimo (apoyo social emocional) probablemente pueda inhibir la intención de quitarse la vida. (Sánchez, Muela y García, 2018).

\section{Comentarios}

La conducta suicida es probablemente uno de los fenómenos en dónde ha sido muy complejo realizar un estudio certero respecto de causas, síntomas o señales que alerten respecto de su manifestación, la característica de multicausal y multidimensional, hacen imposible el siquiera intentar establecer relaciones de causa efecto. De allí que la mayoría de los intentos versan sobre sistematizar información de especialistas, experiencias con personas que han manifestado presentar ideación suicida o el haber desarrollado intentos de suicidio sin haberlo consumado, o en algunos casos a través de entrevistas realizada a cercanos de personas que lograron suicidarse.

El tema está lejos de agotarse y probablemente si las tendencias de los últimos años se mantienen, el suicidio seguirá en aumento desafiando a profesionales clínicos para buscar formas de prevención que sean cada vez más efectivas.

\section{Referencias}

Arce K., \& Arturo, A. A. (2017). Consumo de alcohol, participación de los padres, y otros predictores de suicidio en la juventud boliviana. Gaceta Médica Boliviana, 40(1), 29-34. Recuperado http://search.ebscohost.com/login.aspx?direct=true\&db=lth\&AN=123846833\&lang=es\& site $=$ ehost-live

Ardiles-Irarrázabal, R.\& Alfaro-Robles, P., Díaz-Mancilla, I., \& Martínez-Guzmán, V.(2018). Riesgo de suicidio adolescente en localidades urbanas y rurales por género, región de Coquimbo, Chile. Aquichan, 18(2),160-170. Recuperado de https://aquichan.unisabana.edu.co/index.php/aquichan/article/view/8619

Baader, T., Rojas, C., Molina, J. L., Gotelli, M., Alamo, C., Fierro, C.,... \& Dittus, P. (2014). Diagnóstico de la prevalencia de trastornos de la salud mental en estudiantes universitarios y los factores de riesgo emocionales asociados. Revista chilena de neuropsiquiatría, 52(3), 167-176. doi: 10.4067/S0717-92272014000300004

Barrios-Acosta, M., Ballesteros-Cabrera, M. D. P., Zamora-Vázquez, S., Franco-Agudelo, S., Gutiérrez-Bonilla, M. L., Tatis-Amaya, J., -\& Rodríguez-Melo, C. I. (2017). Universidad y conducta suicida: respuestas y propuestas institucionales, Bogotá 2004-2014. Revista de Salud Pública, 19(2), 153-160. Recuperado de http://www.scielo.org.co/pdf/rsap/v19n2/0124-0064-rsap-19-02-00153.pdf

Borges, G., Orozco, R., Benjet, C., \& Medina-Mora, M. E. (2010). [Suicide and suicidal behaviors in Mexico: Retrospective and current status]. Salud Publica De Mexico, 52(4), 292-304. Recuperado http://search.ebscohost.com/login.aspx?direct=true \&db=mdc\&AN=20657958\&lang=es \& $\underline{\text { site}=\text { ehost-live }}$

Cañón, S. C., Castaño-Castrillón, J. J., Mosquera-Lozano, A. M., Nieto-Angarita, L. M., OrozcoDaza, M., \& Giraldo-Londoño, W. F. (2018). Propuesta de intervención educativa para la prevención de la conducta suicida en adolescentes en la ciudad de Manizales (Colombia). Diversitas: Perspectivas en Psicología, 14(1), 27-40. doi: 10.15332/s17949998.2018.0001.02 
Corona Miranda, B., Hernández Sánchez, M., \& García Pérez, R. M. (2016). Mortalidad por suicidio, factores de riesgos y protectores. Revista Habanera de Ciencias Médicas, 15(1), 90-110. Recuperado de http://scielo.sld.cu/pdf/rhcm/v15n1/rhcm11116.pdf

Echávarri O, Maino M, Fischman R, Morales S, Barros J. (2015) Aumento sostenido del suicidio en Chile: un tema pendiente.Pontificia Universidad Católica de Chile. [visitado 2020 Enero]. Disponible en: https://politicaspublicas.uc.cl/wpcontent/uploads/2015/07/N\%C2\%B0-79-Aumento-sostenido-del-suicidio-en-Chile.pdf

González-Forteza, C., García, G., Medina-Mora, M., \& Sánchez, M. A. (2013). Indicadores psicosociales predictores de ideación suicida en dos generaciones de estudiantes universitarios. Salud mental, 21(3), 1-9. Recuperado de http://www.revistasaludmental.mx/index.php/salud_mental/article/view/702

Hernández Castillo, M. (2016). Uso de programas para la prevención del suicidio. Universidad de Almería, España

Jans, T., Vloet, T. D., Taneli, Y., \& Warnke, A. (2017). Suicidio y conducta autolesiva. Manual de Salud Mental Infantil y Adolescente de la IACAPAP. Ginebra: Asociación Internacional de Psiquiatría del Niño y el Adolescente y Profesiones Afines.

Marín, P., \& Jorka, J. (2018). Programa de Prevención de Conducta Suicida (PRECONSUI) y Autoestima en los adolescentes de la "Institución Educativa Estatal Mariscal Castilla" Distrito de El Tambo-Huancayo, 2013.

Ministerio de Salud de Chile (2019). Departamento de Estadísticas e Información de la Salud. Mortalidad por Suicidio por región y grupos de edad, Chile 2000-2016 . Recuperado de http://www.deis.cl/series-y-graficos-de-mortalidad/

Miranda, I. C. (2009). Ideación suicida en población escolarizada infantil: factores psicológicos asociados. Salud mental, 35, 495-502. Recuperado de http://www.scielo.org.mx/pdf/sm/v32n6/v32n6a7.pdf

Montes-Hidalgo, J., \& Tomás-Sábado, J. (2016). Autoestima, resiliencia, locus de control y riesgo suicida en estudiantes de enfermería. Enfermería clínica, 26(3), 188-193.

Navarrete Betancort, E. M., Herrera Rodriguez, J., \& León Pérez, P. (2019). Los límites de la prevención del suicidio. Revista de La Asociación Española de Neuropsiquiatría, 39(135), 193-214. doi: https://doi.org/10.4321/S0211-57352019000100011

Navarro-Gómez, N. (2017). El suicidio en jóvenes en España: cifras y posibles causas. Análisis de los últimos datos disponibles. Clínica y Salud,28(1), 25-31. doi: 10.1016/j.clysa.2016.11.002

Nieto, Cecilia Marino; Chafey, María I. Jiménez; Caraballo, Carilu Pérez. (2018). Perfil de estudiantes universitarios/as que han tenido intentos suicidas. Revista Puertorriqueña de $\begin{array}{llll}\text { Psicología, 29(2), 364-375. Recuperado de } & \end{array}$ https://dialnet.unirioja.es/descarga/articulo/6773572.pdf

Nova, R., Hamid, A. Y. S., \& Daulima, N. H. C. (2019). La experiencia familiar en el cuidado de clientes con riesgo suicida en Indonesia. Enfermería Global, 18(1), 445-454. doi: $\underline{10.6018 / \text { eglobal.18.1.337751 }}$

Organización Panamericana de la Salud.(2014) Mortalidad por suicidio en las Américas.; Recuperado de https://www.paho.org/hq/dmdocuments/2014/PAHO-Mortalidad-por$\underline{\text { suicidio-final.pdf }}$

Restrepo, J. E., Sánchez, O. A., Vallejo, G. C., Quirama, T. C., Sánchez, Y. O., \& Cardona, P. D. (2018). Depresión y su relación con el consumo desustancias psicoactivas, el estrés académico y la ideación suicida en estudiantes universitarios colombianos. Health \& Addictions/Salud y Drogas, 18(2). 
Romo ML, Kelvin EA. (2016) Impact of bullying victimization on suicide and negative health behaviors among adolescents in Latin America. Rev Panam Salud Publica. 40(5), 347355. Recuperado de https://www.scielosp.org/article/rpsp/2016.v40n5/347-355/

Ruiz, C. P. S., \& Chaves, J. A. O. (2017). Factores de riesgo asociados con la aparición de conductas suicidas en adolescentes. MedUNAB, 20(2), 139-147. doi: $10.29375 / 01237047.2272$

Sanchez, R., Cáceres, H., \& Gómez, D. (2002). Ideación suicida en adolescentes universitarios: prevalencia y factores asociados. Biomédica, 22(2), 407-415. doi: 10.7705/biomedica.v22iSupp2.1189

Sánchez-Teruel, D., Muela-Martínez, J. A., \& García-León, A. (2018). Variables de riesgo y protección relacionadas con la tentativa de suicidio. Revista de Psicopatología y Psicología Clínica, 23(3), 221-229. doi: 10.5944/rppc.vol.23.num.3.2018.19106

Siabato, E., \& Salamanca, Y. (2015). Factores asociados a ideación suicida en universitarios. Psychologia: avances de la disciplina, 9(1), 71-81. Recuperado de http://www.scielo.org.co/pdf/psych/v9n1/v9n1a06.pdf

Silva, D., Valdivia, M., Vicente, B., Arévalo, E., Dapelo, R., \& Soto, C. (2017). Intento de suicidio y factores de riesgo en una muestra de adolescentes escolarizados de Chile. Revista de Psicopatología y Psicología Clínica, 22(1), 33-42. doi: 10.5944/rppc.vol.22.num.1.2017.16170

Stone, D.M., Holland, K.M., Bartholow, B., Crosby, A.E., Davis, S., and Wilkins, N. (2017). Preventing Suicide: A Technical Package of Policies, Programs, and Practices. Atlanta, GA: Centro Nacional para la Prevención y el Control de Lesiones, Centros para el Control y la Prevención de Enfermedades.

Tabares, M. M., Villa, S. R., \& Rendón, S. V. (2019). Relaciones parentofiliales en la infancia. Prevención del comportamiento suicida. Poiésis, (36), 147-163. doi: https://doi.org/10.21501/16920945.3195

U.S. Office of the Surgeon General, National Action Alliance for Suicide Prevention. (2012) National strategy for suicide prevention: goals and objectives for action. Washington, D.C.: HHS; 2012.

Vélez-Pérez, D., Maldonado-Santiago, N., \& Rivera-Lugo, C. I. (2017). Espectro del suicidio en jóvenes universitarios en Puerto Rico. Revista Puertorriqueña de Psicología, 28(1), 34-44. Recuperado de https://dialnet.unirioja.es/servlet/articulo?codigo=5891755

World Health Organization. (2014) Suicide prevention: a global imperative. Geneva, Switzerland: WHO Press. 